grateful for this. The majority of the ePortfolio is good at documenting a basic level of competence. With a sensible reduction in "log entry' volume and a rethink on the content and purpose of the clinical supervisor's report, the ePortfolio could be a fantastic aid to training. That said, what I really value in training is dedicated tutorial time with experienced GPs to talk through challenging cases and difficult scenarios. This apprenticeship-style learning and the passing on of the 'art' of medicine is invaluable and completely irreplaceable by the ePortfolio.

\section{Pete Osborne,}

GP Registrar, Burdwood Surgery, Wheelers Green Way, Thatcham, Berkshire, RG19 4YF. E-mail: peterosbornelanhs.net

Bahia Bal,

Burdwood Surgery, Wheelers Green Way, Thatcham, Berkshire.

\section{REFERENCE}

1. Lakasing E. Formative assessments in medical education: are excessive, and erode the learning and teaching experience. Br J Gen Pract 2013; 63(608): 145.

DOI: 10.3399/bjgp13X669121

\section{Paediatric consultant GP-hotline audit}

It has long been recognised that the telephone is a good way to improve communications between specialists and primary care physicians. ${ }^{1}$ Written communication is often of variable quality and poor educational value. Despite several examples of successful GP hotlines in the literature, it is an area that has never seemed to take off in the NHS. ${ }^{2}$

The paediatric consultants at St Mary's Hospital run a free advice hotline. They receive calls from local GPs between $12-2 \mathrm{pm}$ and give advice relating to queries about children. It is hoped that in addition to providing invaluable telephone support, the hotline helps to streamline or avoid paediatric referrals.

Calls to the hotline were audited over a 1-month period. The purpose of the audit was to analyse the number of GP practices using the hotline, the type of queries the calls were about, the type of advice usually given, whether the hotline avoided subsequent clinic referrals, and whether GPs were satisfied with the service.

We analysed 23 calls from 13 different

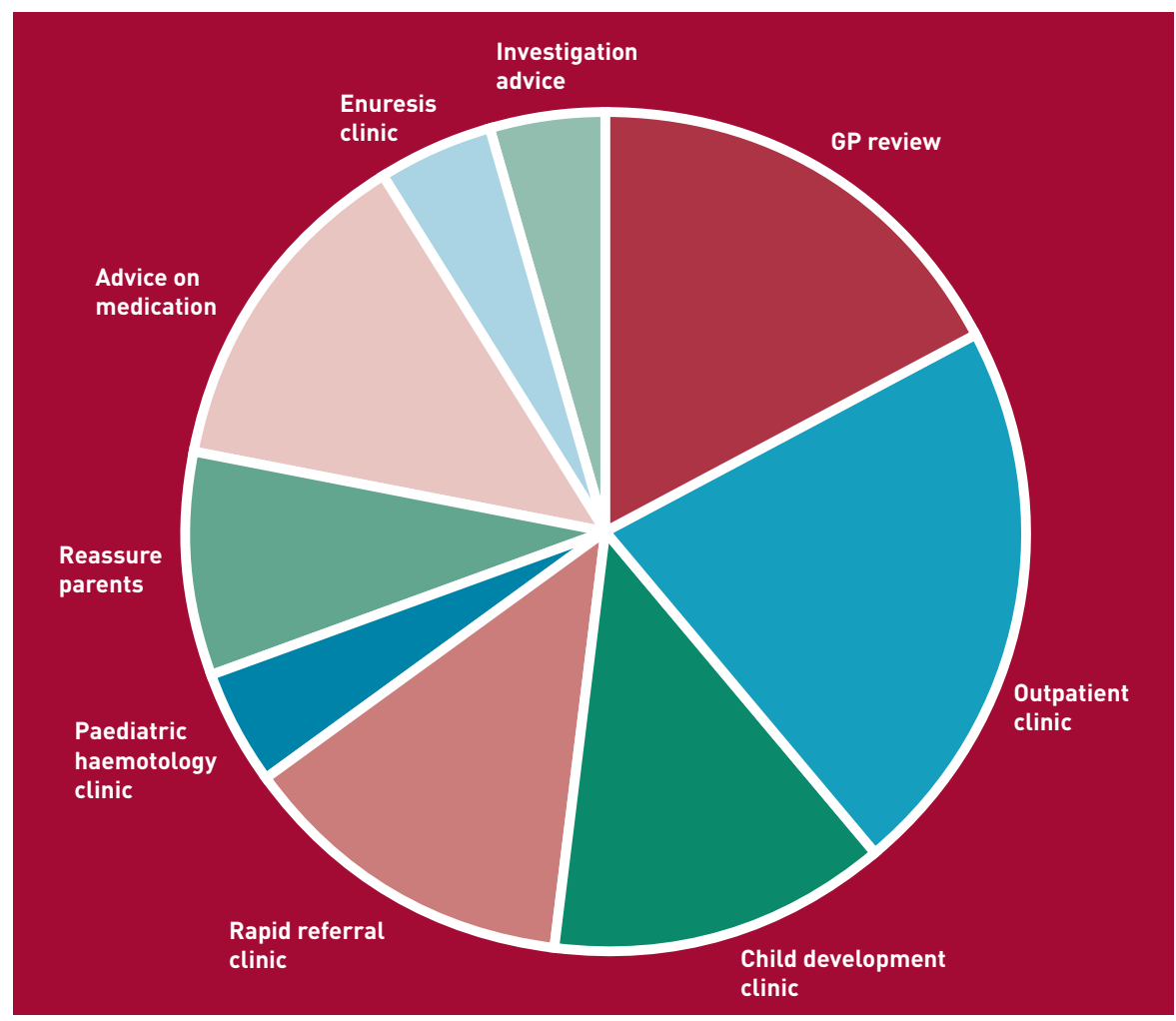

Figure 1. Patient outcomes following hotline calls.

surgeries, which account for around 25\% of the 50 practices that are located within $2 \mathrm{~km}$ of the hospital. Frequent clinical topics discussed included dermatology, gastroenterology, urology, prescribing, and behavioural problems. Some calls avoided referrals and although half of the queries resulted in a referral to paediatric outpatients, these were better directed because of the call. Approximately onethird of the queries were dealt with by reassurance and GP follow-up. (Figure 1.)

Feedback from GPs was universally positive. They liked the time slot, didn't have too much difficulty getting through, and preferred telephone contact over e-mail. Paediatricians thought providing the service was worthwhile and appreciated the opportunity for case discussion and strengthening links with local GPs.

We think that the GP hotline is a successful, useful, and much appreciated service that succeeds in preventing inappropriate referrals and directing necessary referrals to the correct clinic. The service is underused at present and there is a need to publicise it more. A voicemail option would be useful, particularly if usage increases: topics for future GP education would be paediatric prescribing and dermatology.

A new referral template for paediatric outpatients is in development which will have the paediatric hotline number prominently displayed to encourage GPs to consider calling for advice before making a formal referral.

Mando Watson,

The Bays, South Wharf Road, St Mary's Hospital, London, W2 1NY.

E-mail: mando.watsondimperial.nhs.uk

Robert Klaber,

Imperial College Healthcare NHS Trust, St Mary's Hospital, London.

Chiara Haynes,

37 Woodbury Drive, Sutton, Surrey.

Katherine Holt,

Flat 4, 190 Brixton Road, London.

Morag Lenman,

34 Bramfield Road, London.

\section{REFERENCES}

1. Harrison R, Clayton W, Wallace P. Can telemedicine be used to improve communication between primary and secondary care? BMJ 1996; 313(7069): 1377-1380.

2. Roland M, Bewley B. Boneline: evaluation of an initiative to improve communication between specialists and general practitioners. J Public Health Med 1992; 14(3): 307-309.

DOI: 10.3399/bjgp13X669130 